\title{
Desenvolvimento inicial de espécies arbóreas em sistemas agroflorestais no Baixo Amazonas, Pará, Brasil
}

\author{
Initial development of tree species in agroforestry systems in the Lower Amazon, Pará, Brazil \\ Breno Pinto Rayol ${ }^{1 *} \&$ Fabrízia de Oliveira Alvino-Rayol² \\ ${ }^{1}$ Universidade Federal Rural da Amazônia, Belém, PA, Brasil. *Autor para correspondência: bprayol@yahoo.com.br. \\ ${ }^{2}$ Instituto Federal de Educação, Ciência e Tecnologia do Pará, Belém, PA, Brasil.
}

Submissão:05/01/2018 / Aceite: 06/05/2018

\begin{abstract}
RESUMO
Estudos sobre o desenvolvimento inicial de espécies arbóreas em sistemas agroflorestais são importantes, pois fornecem subsídios para o manejo desses agroecossistemas. O objetivo dessa pesquisa foi avaliar a sobrevivência e o crescimento em altura de mudas de espécies arbóreas em sistemas agroflorestais amazônicos submetidos a diferentes formas de preparo da área e manejo. $\mathrm{O}$ experimento foi conduzido em dois sistemas agroflorestais (sucessional e consórcio agroflorestal) localizados no Baixo Amazonas, oeste do estado do Pará. No consórcio agroflorestal comercial, a taxa de sobrevivência das espécies não ultrapassou o valor de $70 \%$. No sistema sucessional a taxa de sobrevivência das espécies arbóreas implantadas foi alta com a maioria variando entre 90 a $100 \%$. 0 estresse hídrico nos períodos mais secos do ano é um fator limitante na adoção de sistemas agroflorestais na região causando mortalidade das mudas. Quanto ao crescimento em altura, as espécies avaliadas obtiveram desempenhos satisfatórios em ambos os sistemas.
\end{abstract}

PALAVRAS-CHAVE: sistema agroflorestal sucessional, consórcio agroflorestal, mudas.

\begin{abstract}
Studies on the initial development of tree species in agroforestry systems are important, since they provide subsidies for the management of these agroecosystems. The objective of this research was to evaluate the survival and height growth of tree species changes in Amazonian agroforestry systems subjected to different forms of area preparation and management. The experiment was carried out in two agroforestry systems (successional and agroforestry consortium) located in Lower Amazon, western Pará state. In the commercial agroforestry consortium, the survival rate of the species didn't exceed $70 \%$. In the successional system, the survival rate of the implanted tree species was high, with most values varying between 90 and $100 \%$. Water stress in the driest periods of the year is a limiting factor in the adoption of agroforestry systems in the region, causing mortality of the seedlings. Regarding the growth in height, the evaluated species obtained satisfactory performances in both systems.
\end{abstract}

KEYWORDS: successional agroforestry system, agroforestry consortium, seedlings.

\section{INTRODUÇÃO}

Inspirada na cobertura permanente que caracteriza a estrutura arbórea da floresta tropical, uma alternativa sustentável promissora de produção primária é a implantação dos diferentes modelos de sistemas agroflorestais (SAF's). Esses sistemas diversificados de produção são caracterizados pelo cultivo simultâneo ou escalonado, no espaço e no tempo, de árvores com espécies agrícolas anuais, plurianuais ou perenes, na presença ou não de animais (NAIR 2007). Nos SAF's é possível combinar os benefícios da produção de alimentos, forragem, energia, madeira, óleos e outros, com os serviços de conservação do solo, manutenção da fertilidade, ciclagem de nutrientes, controle de erosão, fixação de nitrogênio e uma maior estabilidade do microclima na propriedade agrícola (NAIR 2007, BHAGWAT et al. 2008, DE BEENHOUWER et al. 2013, TORRALBA et al. 2016).

Essas tecnologias, quando baseadas em princípios ecológicos, são capazes de promover renda aos agricultores familiares e ainda favorecer o meio ambiente (ABDO et al. 2008, HERMIDA 2015, VASCONCELOS \& BELTRÃO 2018). Existe, portanto, necessidade da realização de estudos que venham 
dar subsídios à implantação e manejo adequado desses sistemas, como por exemplo, estudos sobre as espécies em sistemas agroflorestais (SILVA et al. 2008). Neste contexto, pesquisas que avaliam as espécies que melhor se adaptam a determinada região são importantes, pois além de fornecer subsídios para estratégias de reabilitação e recuperação de áreas degradadas, permitem avaliações qualitativas e quantitativas das áreas sob processo de recuperação que são fortemente perturbadas ao longo do tempo (NAPPO et al. 2004).

Nesta perspectiva, o objetivo do trabalho foi avaliar a sobrevivência e o crescimento em altura de mudas de espécies arbóreas em sistemas agroflorestais amazônicos submetidos a diferentes formas de preparo da área e manejo, com a finalidade de fornecer base nas tomadas de decisões referentes à implantação e gestão desses agroecossistemas.

\section{MATERIAL E MÉTODOS}

O experimento foi conduzido em dois sistemas agroflorestais localizados no Baixo Amazonas, oeste do Estado do Pará. Os SAF's foram implantados em propriedades vizinhas, com histórico e características ambientais semelhantes, localizadas na comunidade de Irurama adjacente a zona urbana de Santarém com coordenadas geográficas $02^{\circ} 29^{\prime} 22,1^{\prime \prime}$ de latitude sul e $054^{\circ} 50^{\prime} 05,3^{\prime \prime}$ de longitude oeste. O clima da região, pela classificação de Köppen, é do tipo Am, com temperatura máxima de 30 a $31^{\circ} \mathrm{C}$ e mínima de 21 a 23 ${ }^{\circ}$ C. A precipitação total anual é em torno de $2000 \mathrm{~mm}$, com distribuição irregular durante os meses. O solo, muito lixiviado, é do tipo Latossolo Amarelo (RODRIGUES et al. 2001).

Foram testados dois sistemas agroflorestais: 1) Agrofloresta sucessional, policultivo de composição diversificada que reúne plantas de variados usos (frutíferas, madeireiras, medicinais, ornamentais etc.) que ocupam variados estratos verticais da vegetação, inclusive a presença de animais (VELOSO 2015); 2) Consórcio agroflorestal, constituído por uma associação de um número limitado de espécies perenes manejadas para fins de comercialização (DUBOIS 1996).

A agrofloresta sucessional foi implantada em agosto de 2010 abrangendo uma área de $1500 \mathrm{~m}^{2}$. No preparo da área não houve o uso do fogo. A implantação foi realizada pelo corte seletivo da vegetação secundária e manutenção da matéria orgânica para incorporação ao solo. Alguns indivíduos arbóreos de médio porte foram deixados na área com o objetivo de evitar a exposição direta à radiação solar dos indivíduos que seriam introduzidos no sistema. Além disso, foram realizadas podas de até $50 \%$ das copas dos indivíduos arbóreos remanescentes.

Durante o experimento o manejo da área foi conduzido por meio de roçada seletiva e poda. Neste sistema, foram utilizadas várias espécies agrícolas de ciclo curto e de ciclo longo. O estrato inferior do sistema foi constituído por curauá (Ananas comosus var. erectifolius [L. B. Smith] Coppus \& Leal), abacaxi (Ananas comosus [L.] Merr.) e feijão (Vigna unguiculata [L.] Walp). O feijão foi introduzido com a finalidade de controlar a competição com a vegetação espontânea. Mudas de espécies agrícolas e florestais também foram implantadas: banana (Musa paradisiaca L.), cacau (Theobroma cacao L.), caju (Anacardium occidentale L.), cupuaçu (Theobroma grandiflorum [Willd. ex Spreng.] K. Schum.), urucum (Bixa orellana L.), cumarú (Dipteryx odorata (Aubl.) Willd.) e paricá (Schizolobium amazonicum Huber ex Ducke).

O segundo sistema foi implantado em fevereiro de 2011 com uma área de $875 \mathrm{~m}^{2}$ constitui um consórcio agroflorestal com as seguintes espécies: mandioca, curauá (Ananas comosus var. erectifolius [L. B. Smith] Coppus \& Leal), pupunha (Bactris gasipaes Kunth), cupuaçu (Theobroma grandiflorum [Willd. ex Spreng.] K. Schum.), mogno (Swietenia macrophylla King) e andiroba (Carapa guianensis Aubl.). O preparo da área deste sistema utilizou as técnicas tradicionais de derruba-e-queima da vegetação secundária e o manejo da vegetação espontânea através da capina não seletiva. Nenhum dos sistemas agroflorestais implantados foram submetidos a sistemas de irrigação.

As espécies com mais de 15 mudas foram selecionadas para mensuração periódica. 0 monitoramento durou 16 meses para o sistema biodiverso e 12 meses para o consórcio agroflorestal. A coleta de dados foi realizada através de avaliações de taxas de sobrevivência e crescimento de altura das mudas com auxílio de uma trena graduada em centímetros.

\section{RESULTADOS E DISCUSSÃO}

No consórcio agroflorestal comercial, a taxa de sobrevivência das espécies não ultrapassou o valor de $70 \%$ (Tabela 1). Embora tenham sido selecionadas espécies nativas e bem adaptadas às condições edafoclimáticas da Amazônia, conforme recomendações de outras pesquisas (JARDIM et al. 2004, RIBEIRO et al. 2014) a taxa de sobrevivência das espécies nesse sistema foi considerada baixa. Uma das principais justificativas que podem explicar esse fato está relacionada com o estresse hídrico que ocorre nos 
meses de menor precipitação pluviométrica na região (agosto a novembro).

Tabela 1. Taxa de sobrevivência das mudas no consórcio agroflorestal comercial no período de 12 meses, Baixo Amazonas, Pará.

Table 1. Survival rate of seedlings in the agroforestry consortium during a 12-month period, Lower Amazon, Pará.

\begin{tabular}{ll}
\hline Nome Comum (Nome científico) & Taxa de Sobrevivência \\
\hline Andiroba (Carapa guianensis) & $66,6 \%$ \\
Mogno (Swietenia macrophylla) & $50,0 \%$ \\
Cupuaçú (Theobroma grandiflorum) & $46,7 \%$ \\
Pupunha (Bactris gasipaes) & $0 \%$ \\
\hline
\end{tabular}

A pupunheira, por exemplo, apesar de ser uma das espécies mais frequentes nos sistemas agroflorestais da Amazônia (SILVA et al. 2008), no presente estudo, sua taxa de sobrevivência no campo foi nula. A pupunheira apresentou decréscimo na taxa de crescimento relativo da altura e do diâmetro do caule no período de menor precipitação pluviométrica em monocultivo no Amazonas (YUYAMA \& SILVA 2003). No estudo sobre a viabilidade agroclimática para o cultivo de pupunheira no Estado do Pará, o município de Santarém, onde foi realizada a presente pesquisa, apresenta potencialidade marginal, o que indica a necessidade de irrigação (BASTOS et al. 2008).

Quanto ao crescimento em altura, as espécies obtiveram desempenhos satisfatórios (Figura 1), porém um pouco inferior quando comparados com outros estudos, realizados em plantio a pleno sol em Manaus, AM com mudas de andiroba (NEVES et al. 1993) e sistema agroflorestal em Santarém, PA com mudas de mogno (MARQUES et al. 1993). Apesar disso, ambas as espécies florestais podem ser recomendadas para o uso em agroecossistemas amazônicos. A andirobeira que possui características morfométricas semelhantes ao mogno (CONDÉ et al. 2013) possui potencial para ser utilizada tanto no enriquecimento de capoeiras, quanto em sistemas agroflorestais (COSTA \& MORAIS 2013). O mogno que também é considerado promissor em sistemas agroflorestais, é uma espécie madeireira de alto valor comercial, e fornece boas condições de sombra e boa produção de liteira (SILVA et al. 2008, CONDÉ et al. 2013).

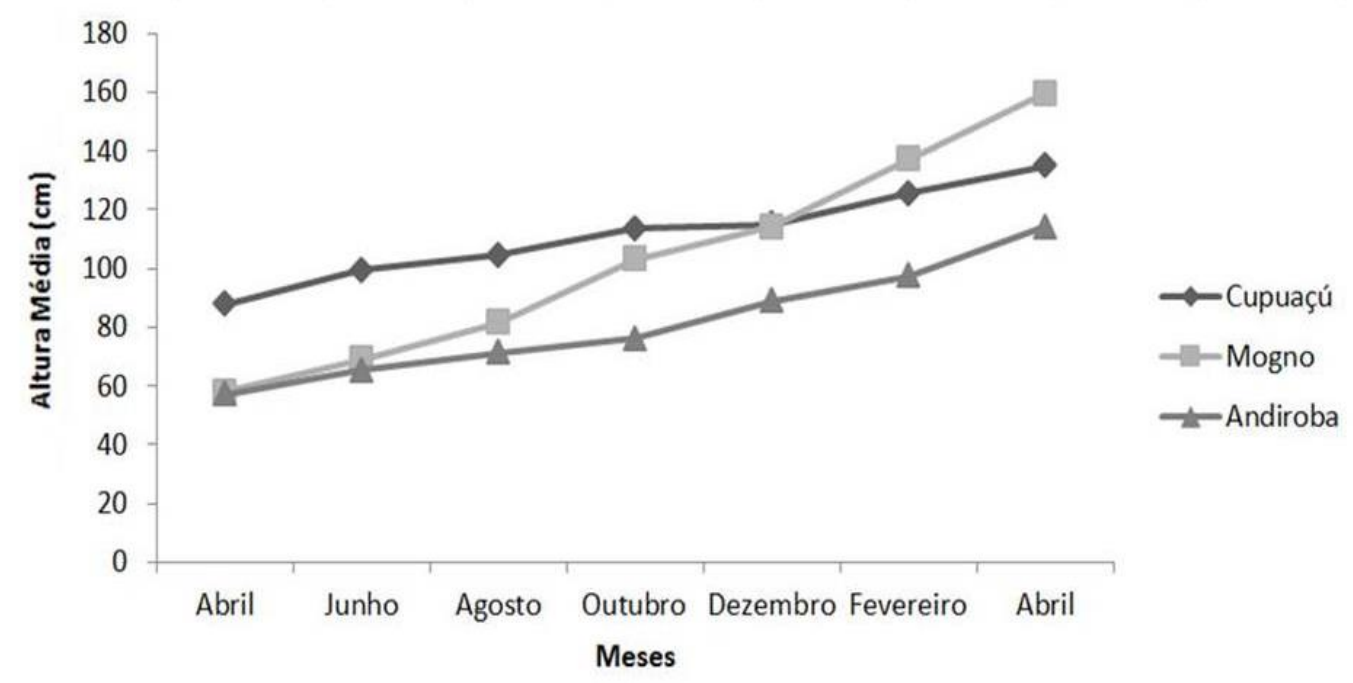

Figura 1. Altura média $(\mathrm{cm})$ das mudas de mogno (Swietenia macrophylla), cupuaçu (Theobroma grandiflorum) e andiroba (Carapa guianensis) em consórcio agroflorestal, Baixo Amazonas, Pará.

Figure 1. Mean height (cm) of mahogany (Swietenia macrophylla), cupuaçu (Theobroma grandiflorum) and andiroba (Carapa guianensis) seedlings in an agroforestry consortium, Lower Amazon, Pará.

Ao contrário do consórcio agroflorestal, na agrofloresta sucessional a taxa de sobrevivência das espécies arbóreas implantadas foi alta com a maioria variando entre 90 a 100\% (Tabela 2). Isso pode estar relacionado com a forma de preparo da área utilizada no sistema biodiverso. Além da vegetação espontânea não ter sido queimada para implantação sistema, as árvores maiores foram deixadas para proteger as mudas da intensidade de radiação solar e da perda excessiva de água. 
Tabela 2. Taxa de sobrevivência das mudas no sistema agroflorestal sucessional no período de 12 meses, Baixo Amazonas, Pará.

Table 2. Survival rate of seedlings in the successional agroforestry system during a 12-month period, Lower Amazon, Pará.

\begin{tabular}{ll}
\hline Nome Comum (Nome científico) & Taxa de Sobrevivência \\
\hline Cacau (Theobroma cacao) & $100 \%$ \\
Cumarú (Dipteryx odorata) & $100 \%$ \\
Cupuaçú (Theobroma grandiflorum) & $95,0 \%$ \\
Paricá (Schizolobium amazonicum) & $94,7 \%$ \\
Banana (Musa paradisiaca) & $91,6 \%$ \\
\hline
\end{tabular}

O estresse hídrico aliado à baixa fertilidade e alta exposição do solo deixam as plantas mais susceptíveis à mortalidade, isso pode causar o abandono do sistema, após a colheita dos componentes agrícolas de ciclo curto, por parte dos produtores ao observarem a alta taxa de mortalidade das espécies arbóreas e arbustivas. Seca e falta de água são alguns dos fatores limitantes na adoção de sistemas agroflorestais citados por agricultores familiares do município de Igarapé-Áçú (VIEIRA et al. 2007). Desta forma, uma alternativa para o uso de sistemas agroflorestais da região é realizar o corte seletivo no momento de limpeza da área e deixar espécies de porte arbóreo e arbustivo remanescentes da vegetação espontânea para proporcionar sombra às mudas pode diminuir a quantidade de perda de água nos meses de menor precipitação pluviométrica. Além disso, o material vegetal oriundo da poda de árvores e arbustos remanescentes pode servir como cobertura morta que além de proporcionar maior proteção e conservação da água no solo, facilita o manejo da vegetação espontânea e contribui com a incorporação de matéria orgânica (AYRES \& RIBEIRO 2010).

As espécies arbóreas introduzidas no sistema sucessional obtiveram bons desempenhos em termos de crescimento em altura durante o período avaliado (Figura 2). O paricá apresentou comportamento semelhante a outros estudos apresentando crescimento rápido e excelente desempenho em consórcios agroflorestais (RIBEIRO et al. 2004, SILVA et al. 2008). A elevada taxa de sobrevivência e o bom desempenho no crescimento em altura e diâmetro do cumarú são indicadores promissores para o seu uso em sistemas agroflorestais (SILVA et al. 2008). Em experimento conduzido em área de produtor familiar localizada as margens da rodovia BR-163, no município de Santarém, sob condições ambientais semelhantes ao do presente estudo (MARQUES et al. 1993), o cumarú associado a outros cultivos (cupuaçu, ingá, banana) apresentou aos 12 meses altura média próxima $(1,30 \mathrm{~m})$ a encontrada no sistema sucessional avaliado nesse estudo $(1,10 \mathrm{~m})$.

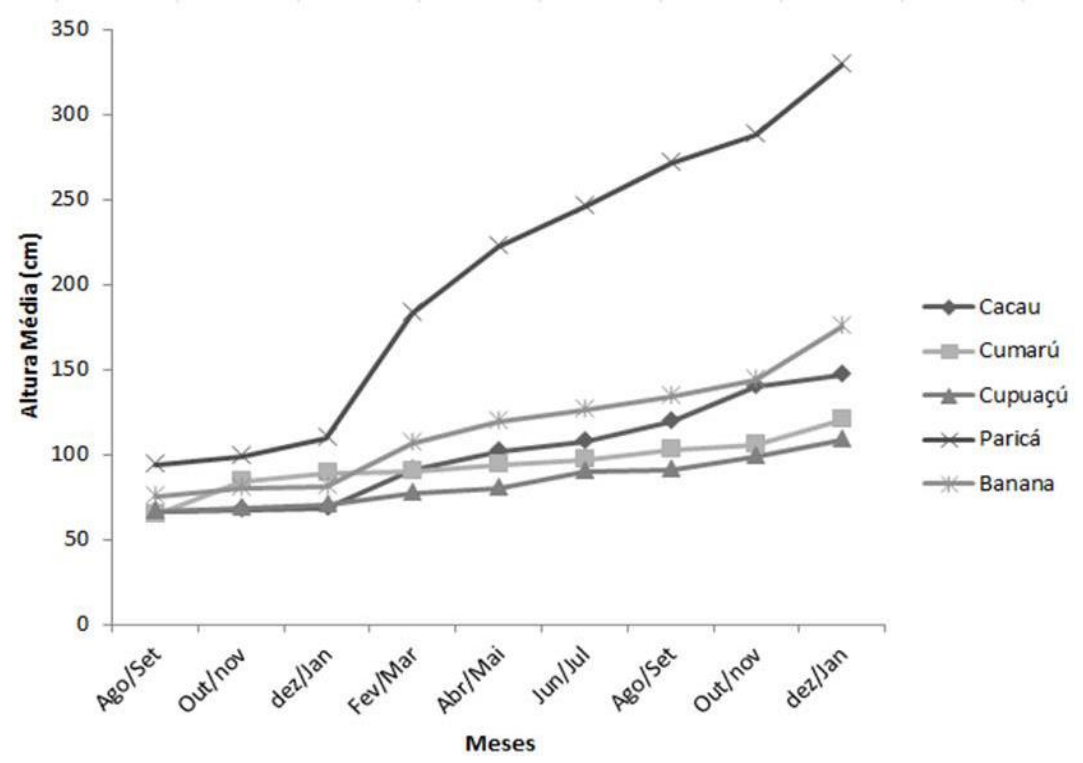

Figura 2. Altura média $(\mathrm{cm})$ das mudas de mogno (Swietenia macrophylla), cupuaçu (Theobroma grandiflorum) e andiroba (Carapa guianensis) em sistema agroflorestal sucessional, Baixo Amazonas, Pará.

Figure 2. Mean height (cm) of mahogany (Swietenia macrophylla), cupuaçu (Theobroma grandiflorum) and andiroba (Carapa guianensis) seedlings in the successional agroforestry system, Lower Amazon, Pará. 
A estratificação de sistemas agroflorestais proporciona condições ambientais favoráveis para o desenvolvimento de culturas como o cupuaçu e cacau (RIBEIRO et al. 2004, ABDO et al. 2008) que são espécies muito utilizadas em sistemas agroflorestais na Amazônia, como, por exemplo, nos sistemas silviagrícolas de Tomé-Açú (BOLFE \& BATISTELLA 2011). O sombreamento das bananeiras facilita a criação de um microclima apropriado para o crescimento nas fases iniciais do desenvolvimento do cupuaçu (RIBEIRO et al. 2004). Além disso, as bananeiras podem contribuir com a diminuição do ataque de Hypsipyla grandella Zeller em plantas de mogno, tal como observado em sistema agroflorestal no município de Santarém (MARQUES et al. 1993).

Além disso, o sistema agroflorestal sucessional implantado se destaca pela sua diversidade vegetal. Dentre os principais produtos pode-se citar: banana, cupuaçu, abacaxi, curauá urucum. Essa diversidade proporciona uma maior diversificação da produção, bem como melhorias na qualidade física, química e biológica do solo, melhoria da capacidade produtiva da terra e otimização do uso de recursos naturais (ABDO et al. 2008, AYRES \& RIBEIRO 2010).

\section{CONCLUSÃO}

O desenvolvimento inicial das mudas em sistemas agroflorestais está diretamente relacionado com o preparo, limpeza e manejo da área. Apesar do crescimento satisfatório, em termos de altura, das mudas nos dois sistemas testados, a sobrevivência das espécies foi maior no sistema agroflorestal sucessional.

Além da função de proteção das mudas introduzidas, a variedade de espécies ocupando os diferentes estratos na estrutura vertical de um sistema agroflorestal sucessional contribui com o melhor aproveitamento do espaço, bem como possibilita maior diversificação de produtos para autoconsumo e/ou comercialização em diferentes épocas do ano.

\section{AGRADECIMENTOS}

À Fundação Amazônia Paraense de Amparo à Pesquisa (FAPESPA) pelo apoio financeiro. Aos agricultores e agricultoras familiares pela cordialidade com que nos receberam em suas propriedades e aos revisores pelas sugestões e contribuições que enriqueceram o presente trabalho.

\section{REFERÊNCIAS}

ABDO MTVN et al. 2008. Sistemas agroflorestais e agricultura familiar: uma parceria interessante. Revista Tecnologia \& Inovação Agropecuária 1: 50-59.

AYRES ECB \& RIBEIRO AEM 2010. Inovações agroecológicas no Nordeste de Minas Gerais: o caso dos sistemas agroflorestais na agricultura familiar do alto Jequitinhonha. Organizações Rurais \& Agroindustriais 12: 334-354.

BASTOS TX et al. 2008. Zoneamento agroclimático para a cultura da pupunheira para a produção de palmito no Estado do Pará. Belém: EMBRAPA. 24p. (Documentos 318).

BHAGWAT SA et al. 2008. Agroforestry: a refuge for tropical biodiversity? Trends in Ecology and Evolution 23: 261-267.

BOLFE EL \& BATISTELLA M. 2011. Análise florística e estrutural de sistemas silviagrícolas em Tomé-Açu, Pará.

Pesquisa Agropecuária Brasileira 46: 1139-1147.

CONDÉ TM et al. 2013. Morfometria de quatro espécies florestais em sistemas agroflorestais no munícipio de Porto Velho, Rondônia. Revista Agro@mbiente On-line 7: 18-27.

COSTA JR \& MORAIS RR. 2013. Carapa guianensis Aubl. (Andirobeira) em Sistemas Agroflorestais. Manaus: EMBRAPA. 28p. (Documentos 112).

DE BEENHOUWER M et al. 2013. A global meta-analysis of the biodiversity and ecosystem service benefits of coffee and cacao agroforestry. Agriculture, Ecosystems \& Environment 175: 1-7.

DUBOIS JCL. 1996. Manual agroflorestal para a Amazônia. Rio de Janeiro: REBRAF. 228p.

HERMIDA GC. 2015. Agroforesteria periurbana una opción para la producción sustentable en los alrededores de Buenos Aires. Scientia Agroalimentaria 2: 7-17.

JARDIM FCS et al. 2004. Avaliação preliminar de sistema agroflorestal no Projeto Água Verde, ALBRÁS, Barcarena, Pará-I. Revista de Ciências Agrárias 41: 25-46.

MARQUES LCT et al. 1993. Alternativa agroflorestal para pequenos produtores agrícolas em áreas de terra firme do município de Santarém, Pará. Belém: EMBRAPA. 18p. (Boletim de Pesquisa 147).

NAIR PK. 2007. The coming of age of agroforestry. Journal of the Science of Food and Agriculture 87: 1613-1619.

NAPPO ME et al. 2004. Dinâmica da estrutura fitossociológica da Regeneração Natural em sub-bosque de Mimosa scabrella Bentham em área minerada, em Poços de Caldas, MG. Revista Árvore 28: 811-829.

NEVES EJM et al. 1993. Comportamento de espécies florestais a pleno sol e em linhas de enriquecimento em Manaus-

AM. In: 7 Congresso Florestal Panamericano; 1 Congresso Florestal Brasileiro. Anais... São Paulo: SBEF. p.756.

RIBEIRO GD et al. 2004. Avaliação preliminar de sistema agroflorestal no Projeto Água Verde, ALBRÁS, Barcarena,

Pará-II. Revista de Ciências Agrárias 41: 49-72.

RODRIGUES TE et al. 2001. Caracterização dos solos da área do planalto de Belterra, município de Santarém, Estado 
do Pará. Belém: Embrapa Amazônia Oriental. 55p. (Documentos 115).

SILVA PTE et al. 2008. Principais espécies florestais utilizadas em sistemas agroflorestais na Amazônia. Revista de Ciências Agrárias 49: 49-72.

TORRALBA $M$ et al. 2016. Do European agroforestry systems enhance biodiversity and ecosystem services? A metaanalysis. Agriculture, Ecosystems \& Environment 230: 150-161.

VASCONCELLOS RC \& BELTRÃO NES. 2018. Avaliação de prestação de serviços ecossistêmicos em sistemas agroflorestais através de indicadores ambientais. Interações (Campo Grande) 19: 209-220.

VELOSO AS. 2015. Implantação do sistema agroflorestal sucessional para conservação de sete nascentes em área degradada por pastagem. Revista Terceiro Incluído 5: 428-451.

VIEIRA TA et al. 2011. Adoção de sistemas agroflorestais na agricultura familiar, em Igarapé-Açu, Pará, Brasil. Revista de Ciências Agrárias 47: 9-22.

YUYAMA K \& SILVA FMS. 2003. Desenvolvimento inicial da pupunheira em monocultivo e intercalado com culturas anuais. Horticultura Brasileira 21: 15-19. 